\title{
A Prospective Study Investigating the Efficacy and Toxicity of Definitive ChemoRadadiation and ImmunOtherapy (CRIO) in Locally and/or Regionally Advanced Cutaneous Squamous Cell Carcinoma.
}

Charles Lin ( $\nabla$ charlesymlin@gmail.com )

Royal Brisbane and Women's Hospital https://orcid.org/0000-0001-5589-4775

Trishna Ballah

Royal Brisbane and Women's Hospital

Michelle Nottage

Royal Brisbane and Women's Hospital

Karen Hay

QIMR Berghofer Medical Research Institute

Benjamin Chua

Royal Brisbane and Women's Hospital

Lizbeth Kenny

Royal Brisbane and Women's Hospital

Paul Thomas

Royal Brisbane and Women's Hospital

Michele WL Teng

QIMR Berghofer Medical Research Institute

Jacqui Keller

Royal Brisbane and Women's Hospital

Trang Le

Royal Brisbane and Women's Hospital

Jennifer Edmunds

Royal Brisbane and Women's Hospital

Brett GM Hughes

Royal Brisbane and Women's Hospital

Study protocol

Keywords: Skin cancer, SCC, curative, radiotherapy, immunotherapy

Posted Date: October 9th, 2020 
DOI: https://doi.org/10.21203/rs.3.rs-88311/v1

License: (c) (1) This work is licensed under a Creative Commons Attribution 4.0 International License. Read Full License

Version of Record: A version of this preprint was published at Radiation Oncology on April 9th, 2021. See the published version at https://doi.org/10.1186/s13014-021-01795-5. 


\section{Abstract}

Background: Patients with unresectable advanced cutaneous squamous cell carcinoma (CSCC) are generally treated with palliative intent. Immune checkpoint blockade has significant activity in the palliative setting in patients with recurrent or metastatic CSCC. This single arm phase 2 prospective study aims to investigate the combination of curative intent chemoradiation and durvalumab (anti-PD-L1 checkpoint inhibitor) for this patient cohort. Our hypothesis is that $>70 \%$ of patients with locally-advanced primary disease or regional metastases can be safely treated for cure using ChemoRadiation and ImmunOtherapy (CRIO) compared to the null hypothesis of $\leq 50 \%$.

Methods: Patients with unresectable locally and or regionally advanced pathologically confirmed cSCC deemed suitable for CRIO by consensus of the Head and Neck Multidisciplinary meeting will be eligible. We aim to accrue a total of 15 patients. The co-primary endpoints of CRIO will be the safety of treatment and the complete response rate. Secondary endpoints will include overall survival, progression free survival, and locoregional control. Translational research endpoints including biomarkers will also be explored utilising multiplex immunohistochemistry on tumour biopsy samples obtained prior to commencing treatment and during treatment (week 2). In addition, the utility of CXCR-4 PET scan will be explored.

Discussion: CRIO is a novel trial evaluating the combination of curative intent chemoradiotherapy with concurrent durvalumab for patients with inoperable locally advanced cSCC.

Trial registration Trial registered with the Australian New Zealand Clinical Trial Registry (ACTRN12618001573246)

\section{Administrative Information}

\section{Note}

the numbers in curly brackets in this protocol refer to SPIRIT checklist item numbers. The order of the items has been modified to group similar items [see http://www.equator-network.org/reportingguidelines/spirit-2013-statement-defining-standard-protocol-items-for-clinical-trials/]. 


\begin{tabular}{|ll|}
\hline Title $\{1\}$ & $\begin{array}{l}\text { A prospective study investigating the efficacy and toxicity of definitive } \\
\text { ChemoRadiation and ImmunOtherapy (CRIO) in locally and/or regionally } \\
\text { advanced cutaneous squamous cell carcinoma }\end{array}$ \\
\hline $\begin{array}{l}\text { Trial registration }\{2 \mathrm{a} \\
\text { and } 2 \mathrm{~b}\} .\end{array}$ & $\begin{array}{l}\text { Australian and New Zealand Clinical Trial Registry (ANZCTR) } \\
\text { ACTRN12618001573246 }\end{array}$ \\
\hline Protocol version $\{3\}$ & Version 1, 3 September 2018 \\
\hline Funding $\{4\}$ & Funded by Astra Zeneca \\
\hline Author details $\{5 \mathrm{a}\}$ & $\begin{array}{l}\text { Charles Lin, Trishna Ballah, Michelle Nottage, Karen Hay, Karen Hay, Benjamin } \\
\text { Chua, Lizbeth Kenny, Paul Thomas, Michele WL Teng, Jacqui Keller, Trang Le, } \\
\text { Jennifer Edmunds, Brett G. M. Hughes }\end{array}$ \\
\hline $\begin{array}{l}\text { Name and contact } \\
\text { information for the } \\
\text { trial sponsor }\{5 \mathrm{~b}\}\end{array}$ & $\begin{array}{l}\text { Metro North Hospital and Health Service, Queensland, Australia. } \\
\text { Address: Royal Brisbane and Women's Hospital, 7 Butterfield St, Herston QLD } \\
\text { 4029, Australia }\end{array}$ \\
\hline Role of sponsor $\{5 \mathrm{c}\}$ & $\begin{array}{l}\text { Overall responsibility to initiate and conduct the study. The Institution has } \\
\text { designated the Principal Investigator the day-to-day conduct of the study and } \\
\text { to lead, train and supervise study site staff. } \\
\text { Astra Zeneca will collaborate with the institution to support the study as per } \\
\text { the terms in the research agreement. }\end{array}$ \\
\hline
\end{tabular}

\section{Introduction}

\section{Background and rationale $\{6 a\}$}

Cutaneous squamous cell carcinoma (CSCC) of the skin is the second most common skin cancer (after basal cell carcinoma). Lifestyle changes in the last 50 years have led to increased exposure to sunlight provoking a sharp rise in its incidence worldwide, with Queensland, a state in Australia, having the highest incidence of cutaneous skin malignancies in the world [1]. Early CSCC is often curable with local therapy. Metastases are rare, but spread to regional lymph nodes or more distant sites occurs in $5-10 \%$ of patients and confers a poor outcome [2-4]. A presentation with locally advanced primary disease or regional nodal metastases is not uncommon and patients face the grim prospect of morbid surgery or uncontrolled loco-regional disease affecting critical areas in the head and neck. Current standard of treatment is surgery followed by adjuvant radiotherapy. However, a proportion of patients cannot undergo surgery for a variety of reasons. In these cases, definitive radiation alone can be offered, but it is anticipated that locoregional control and cure rates would be low with single modality treatment [5].

The use of concurrent chemotherapy with radiotherapy for CSCC in the definitive setting has been investigated in several retrospective series, with most utilising cisplatin-based chemotherapy. Results indicated partial response between 17-54\% and complete responses in 17-43\% [6-9]. Our institution has published the only prospective study of definitive chemoradiation in this setting. This demonstrates a $50 \%$ rate of complete response with combined radiotherapy and concurrent cisplatin/carboplatin [10]. 
Complete responders after definitive chemoradiation rarely recur in the irradiated area. It is therefore logical to consider adding an agent to improve on the $50 \%$ complete response rate.

CSCC has several clinical and biological factors which suggests it is appropriate for the clinical study of inhibition of the Programmed Death-1/Programmed Death- Ligand 1 (PD-1/PD-L1) immune check point: high mutation burden [11] presence of tumour-infiltrating-lymphocytes [11-14], association with immunosuppression as a risk factor [15], evidence of direct immunosuppressive effects of UV radiation [16], and some clinical efficacy with interferon 2a-based treatment [17]. The presence of high mutation burden is also a common characteristics of other solid tumours for which inhibition of the PD-1/PD-L1 axis has been associated with therapeutic efficacy, including melanoma, non-small cell lung cancer, and urothelial cancer [18].

The use of PD-1/PD-L1 immune check point inhibitor is generally well tolerated and publications of prospective phase 2 studies [19-20] on cemiplimab have shown impressive response of patients with recurrent advanced/metastatic cSCC being sensitive to PD-1 blockade. The survival benefit of adding immunotherapy to chemoradiation is also evident in the setting of locally advanced non-small cell lung cancer as shown in a randomized controlled trial [21]. Durvalumab (MEDI4736) is a human monoclonal antibody of the immunoglobulin G-1 kappa subclass that inhibits binding of PD-L1. As Durvalumab (MEDI4736) is an engineered monoclonal antibody, it does not induce antibody-dependent cellular cytotoxicity or complement-dependent cytotoxicity.

\section{Objectives $\{7\}$}

The Primary Objectives was to assess the safety of treatment (toxicities and adverse events) and to assess the rate of complete response.

The secondary objective was to assess overall survival, progression free survival, and locoregional control. In addition, the correlation of CXCR-4 uptake on PET scan (see below in Study setting) to overall survival, progression free survival, and locoregional control.

\section{Trial Design \{8\}}

CRIO is a single arm, non-blinded, prospective, phase II study in a single Australian academic hospital

\section{Methods: Participants, Interventions And Outcomes}

\section{Participants, interventions and outcomes}

\section{Study setting $\{9\}$}

This trial is a single arm, phase II, prospective study conducted in a single Australian academic hospital in Queensland. Data will be collected at the hospital where patients are being accrued.

\section{Translational studies}


Adenosine is generated in response to proinflammatory stimuli such as cellular stress initiated by hypoxia or ischemia [22-23]. Release of extracellular ATP undergoes conversion to AMP by the enzyme CD39 and subsequent dephosphorylation of AMP to adenosine is catalyzed by CD73. Adenosine signaling, particularly via the A2A adenosine receptor, potently reduces effector functions of cytotoxic lymphocytes (CD8 $+T$ cells and NK cells) while also promoting recruitment and polarization of immunosuppressive cell types, including myeloid-derived suppressor cells and T regulatory cells [24]. Previously, we and other have identified that targeting adenosine generation by blockade of the ectonucleotidase CD73 or downstream A2AR inhibition enhances tumour control and anti-metastatic activity [25-28]. In addition, therapeutic approaches targeting the adenosinergic pathway alongside immune checkpoint blockade and chemotherapies display enhanced anti-tumour efficacy in combination [22, 29-31]. Importantly, targeting adenosine in solid tumours using anti-CD73 (NCT02503774) or A2A adenosine receptor antagonism (NCT02403193 and NCT02655822) has entered clinical trials.

We previously demonstrated demonstrating that in more advanced clinical stage, CD73 expression is increased in cutaneous melanoma patients who did not receive any immunotherapy or BRAF/MEKi. In this present study, we aim to evaluate if the adenosinergic pathway is present in CSCC and its relationship with the PD-1/PD-L1 pathway. We will utilize multiplexed immunohistochemistry to evaluate the expression of CD73, CD39, PD-1, PD-L1 on tumour, endothelial or immune cells on tumour biopsies to examine cellular aspects of response. These biopsies will be obtained at baseline and if feasible, biopsies will also be obtained at week 2 while patients are on treatment. Furthermore, all patients will have serial blood sampled (baseline, weeks 3, 13, 21 and 37) for retrospective immunological and molecular studies should funding be obtained. This will include FACS analysis for innate and adaptive immune cell, and their expression of activatory and inhibitory receptors. mRNA can be isolated and immune signatures assessed using either Nanostring panel or RNAsEq. The clonality of T-cells can be examined by sequencing the TCR using Illumina-based next generation sequencing.

Results could provide the first proof-of-principle clinical evidence of the prognostic relevance of the density of immune cell infiltration, and presence of the adenosinergic pathway in cSCC.

\section{CXCR4 PET Scan as a pilot sub-study}

CXCR-4 is a chemokine receptor identified to promote the metastasis of a variety of cancer, including mucosal head and neck SCC. The over-expression of the CXCL12/CXCR4 chemokine system is likely to play a role in several tumour promoting effects including metastasis and resistance to treatment [32]. Promising human trial results for antibodies targeting other T-cell immunosuppressive mechanisms (e.g. immune checkpoints PD-1/PD-L1 and CTLA-4) have been reported for several cancers. A recent preclinical study demonstrated that targeting CXCR4 through inhibitory antibodies, in combination with anti-PD-L1, caused tumour regression in a mouse model of cancer which is resistant to PD-1 and CTLA-4 blockade [33]. This provides a good rationale for exploring the relationship between CXCR4 and immunotherapy, of which little is known in human SCC. 
At the Herston Imaging and Research Facility within our institution, we have access to a novel Positron Emission Tomography (PET) tracer, 68 Ga-CPCR4-2 (68 Ga-Pentixafor) which binds to CXCR4 receptors. It is hypothesized that cutaneous SCC with higher CXCR4 uptake would be at greater risk of nodal and distant metastases. As a pilot sub-study, we aim to explore the utility of CXCR4 in predicting the response to CRIO. Participation in the CXCR4 pilot sub-study is optional and all patients enrolled in the CRIO study will be treated and followed up the same way. The information obtained from the CXCR4 pilot study is hypothesis generating given the likely small patient number. If a trend is detected in its predictive utility, this may lead to the use of CXCR4 PET for CSCC in future trials.

\section{Eligibility criteria $\{10\}$}

Inclusion criteria were as follows: adult patients presenting with pathologically confirmed unresectable cSCC with locally-advanced disease at the primary site with or without metastases to the nodal regions, or advanced regional lymph node metastases of presumed skin origin, or unresectable perineural disease; Eastern Cooperative Oncology Group performance status 0-2; and assessable disease. Patients with bulky disease are assessed using RECIST criteria. Patients with perineural disease must have disease visible on imaging or biopsy-proven disease.

Exclusion Criteria include: Immunosuppression secondary to blood disorders or immunosuppressant for organ transplants or human immunodeficiency virus (HIV); active infection with Hepatitis B or C virus; active uncontrolled bleeding; previous chemotherapy and/or radiotherapy to the head and neck region which would preclude re-treatment; contra-indication for radiotherapy or chemotherapy; claustrophobia; previous treatment with a PD1 or PD-L1 inhibitor; receipt of anticancer therapy 60 days prior to the first dose of study drug; use of immunosuppressive medication within 14 days before the first dose of Durvalumab (MEDI4736); any unresolved toxicity NCI CTCAE v 4.03 Grade $\geq 2$ from previous anticancer therapy; history of allogenic organ transplantation; active or prior documented autoimmune or inflammatory disorders and receipt of recent live attenuated vaccine

\section{Who will take informed consent? $\{26 \mathrm{a}\}$}

The informed consent process will be carried out either by the treating clinician or an appropriately trained member of the site research team. A treating clinician will provide eligible patients with the information and consent form for the trial. Patients will be given time to read the information and will be provided opportunities to clarify any concerns or ask questions prior to providing informed consent.

\section{Additional consent provisions for collection and use of participant data and biological specimens \{26b\}}

Patients will be offered participation in two optional sub-studies. Patients will be asked to consent to participate in a sub-study which investigates the predictive power of a novel PET scan using a novel CXCR4 tracer. Such a tracer has never been used in patients with cSCC in Australia. The result of this PET scan will not impact on the treatment the patient receives, though the results obtained from this substudy may be used to adjust treatment for future patients suffering from a similar condition. The CXCR4 PET scans will be performed at baseline (prior to starting treatment) AND during week 3 of the treatment. 
Patients will also be asked for a repeat core biopsy of the skin cancer or enlarged lymph node during the second week of treatment. Additional consent for this second biopsy will need to be obtained. The purpose is to identify any potential biomarkers in the biopsy specimen which may predict treatment response.

Participation in both sub-studies is optional. Patients who decline to participate in this sub-study will receive the same treatment as other study participants. Interventions

\section{Explanation for the choice of comparators $\{6 \mathrm{~b}\}$}

Not applicable. This is a single arm study without a comparator.

\section{Intervention description $\{11$ a $\}$}

\section{Chemotherapy}

Platinum-based chemotherapy will commence on either day 1-3 of the radiation and preferably repeated on the same day of each week. A maximum of 7 weekly doses will be given. In cases where the chemotherapy is delayed or ceased, the radiotherapy will continue according to protocol. The primary chemotherapy regimen is cisplatin $40 \mathrm{mg} / \mathrm{m}^{2} /$ week. Carboplatin may be used for patients with renal or cardiac or hearing impairment, at the discretion of the treating physician. Full blood counts, ELFT, magnesium, and renal function tests must be performed within 48 hours prior to each weekly chemotherapy dose.

\section{Immunotherapy}

Durvalumab will be supplied by AstraZeneca as a 500-mg vial solution for infusion after dilution. The solution contains $50 \mathrm{mg} / \mathrm{mL}$ durvalumab, $26 \mathrm{mM}$ histidine/histidine hydrochloride, $275 \mathrm{mM}$ trehalose dihydrate, and $0.02 \%$ weight/volume $(\mathrm{w} / \mathrm{v})$ polysorbate 80 ; it has a $\mathrm{pH}$ of 6.0 . The nominal fill volume is $10.0 \mathrm{~mL}$. Investigational product vials are stored at $2{ }^{\circ} \mathrm{C}$ to $8{ }^{\circ} \mathrm{C}\left(36^{\circ} \mathrm{F}\right.$ to $\left.46^{\circ} \mathrm{F}\right)$ and must not be frozen. Drug product should be kept in secondary packaging until use to prevent excessive light exposure.

- Concurrent phase: administered every 4 weeks during chemoradiation (weeks 1 and 5).

- Adjuvant phase: same dose and frequency as concurrent phase in weeks 9,13 and 17. Up to 6 additional doses until the disease status can be determined.

- Fixed dosing of $1500 \mathrm{mg}$ every 4 weeks (approximate $20 \mathrm{mg} / \mathrm{kg}$ every 4 weeks)

- 3 Radiotherapy

Intensity Modulated Radiotherapy (IMRT) should be utilised on all patients in the study cohort.

\subsection{Target volumes and Doses:}

\section{Locally advanced disease without nodal metastasis}

Gross disease at the primary site: Gross Tumour Volume (GTV) should include all the imageable/palpable extent of the gross disease. In-transit dermal metastases, if evident, are to be included in the GTV. A 
recommended $5 \mathrm{~mm}$ margin is to be added to the GTV to form the Clinical Target Volume (CTV) to include any microscopic disease. CTV will then be expanded by $5 \mathrm{~mm}$ to form the Planning Target Volume (PTV), to account for any setup error and patient movement. Where possible, the PTV is to receive a total dose of $70 \mathrm{~Gy}$ in 35 fractions over 7 weeks as per ICRU 83 [34]. The dose variation across the PTV should not exceed $+7 \%$ and $-5 \%$ of the prescription point (ICRU reference point dose). Where the location of the PTV precludes delivery of $70 \mathrm{~Gy}$ due to surrounding normal tissue constraint, a lower dose of $66 \mathrm{~Gy}$ in 33 fractions over 6.5 weeks is acceptable.

\section{Prophylactic nodal irradiation:}

All attempts are to be made to include the first lymphatic draining echelon in the radiotherapy field (e.g. to include the parotid region when the primary disease is located at the temple). As these sites may harbour subclinical/microscopic disease, a total dose of $54 \mathrm{~Gy}$ in 35 fractions over 7 weeks, as per ICRU 83, is to be delivered. The dose variation across the PTV should not exceed $+7 \%$ and $-5 \%$ of the prescription point (ICRU reference point dose). The level of cervical lymph nodes is to be defined as per Gregoire et al [35].

\section{Locally advanced disease or occult primary disease with nodal metastases Gross disease at the primary site and macroscopically involved nodes:}

GTV should include all the imageable/palpable extent of the disease at the primary (skin) site and the macroscopically involved lymph nodes. In-transit dermal metastases, if evident, are to be included in the GTV. A recommended $5 \mathrm{~mm}$ margin is to be added to the GTV to form the CTV to include any microscopic disease. CTV will then be expanded by $5 \mathrm{~mm}$ to form the PTV, to account for any setup error and patient movement. Where possible, the PTV is to receive a total dose of $70 \mathrm{~Gy}$ in 35 fractions over 7 weeks as per ICRU 83. The dose variation across the PTV should not exceed $+7 \%$ and $-5 \%$ of the prescription point (ICRU reference point dose). Where the location of the PTV precludes delivery of 70 Gy due to surrounding normal tissue constraint, a lower dose of $66 \mathrm{~Gy}$ in 33 fractions over 6.5 weeks is acceptable.

\section{Prophylactic nodal irradiation:}

The subsequent lymphatic draining echelons next to the involved nodal region should be included in the radiotherapy field (e.g. to include level II to III ipsilateral cervical lymph nodes when parotid region is clinically involved). As these sites may harbour subclinical/microscopic disease, a total dose of $54 \mathrm{~Gy}$ in 35 fractions over 7 weeks, as per ICRU 83, is to be delivered. The level of cervical lymph nodes is to be defined as per Gregoire et al [35].

\section{Large nerve perineural spread (PNS)}

The extent of PNS can be defined on 3T Magnetic Resonance Imaging (MRI) and/or clinical examinations. The most commonly involved cranial nerves in the setting of head and neck cutaneous SCC are the fifth (Trigeminal) and the seventh (Facial) cranial nerves. PNS are to be encompassed in the CTV and a $5 \mathrm{~mm}$ margin should be added to form the PTV. However, a smaller margin of $<5 \mathrm{~mm}$ can be accepted if vital organs at risk are immediately adjacent to the PNS. Where possible, the involved nerve(s) 
should receive $\geq 66 \mathrm{~Gy}$ in 33 fractions. Where vital organs at risk (e.g. optic chiasm or brain stem) are immediately adjacent to the PNS, a lower dose between 54 Gy -66Gy in 2 Gy per fraction can be accepted.

The extent of elective nerve irradiation in continuity with the known PNS disease (Based on MRI and/or clinical examinations) is to be determined by the treating Radiation Oncologist. For example, a patient with evidence of right infraorbital nerve involvement may also receive elective radiotherapy to the proximal segment of V2 up to the trigeminal ganglion. The dose of elective nerve irradiation is generally between 50-60Gy, respecting the surrounding vital organs at risk.

\section{Criteria for discontinuing or modifying allocated interventions $\{11 \mathrm{~b}\}$}

Intervention safety monitoring and assessment Adverse events (AE), defined as any untoward medical occurrence(s) in a trial participant regardless of causality with trial interventions, will be systematically monitored and recorded. These will be classified and graded according to the National Cancer Institute Common Terminology Criteria for Adverse Events version 4.03 (NCI CTCAE v4.03).

Serious AEs (SAE) will be reported to the appropriate ethics committees and competent authorities as well as the study safety committee. A suspected unexpected serious adverse reaction (SUSAR), which is an unexpected SAE related to the intervention, will additionally be reported to the drug manufacturer. If the $\mathrm{AE}$ is deemed by the investigator to have been caused, or probably caused, by the investigational treatment (denosumab), this will be labelled a TRAE (treatment-related adverse event). Study participants will be reviewed clinically for the presence of AEs by the site investigator prior to each cycle of chemotherapy or immunotherapy. Blood tests, including complete blood count, liver and renal function tests, electrolytes, thyroid function tests and serum cortisol will be reviewed at these visits. Immunerelated adverse events are managed following algorithms as compiled by expert groups or as available in the product information. Re-challenge after a suspected TRAE is permitted provided symptoms resolve to an appropriate level to meet criteria to resume treatment and do not meet any of the permanent discontinuation criteria (such as grade 4 and select grade 3 toxicities). Dose reductions and dose escalations are not permitted. An independent data safety monitoring committee will monitor the conduct and safety of the trial during recruitment.

\section{Permanent Discontinuation of Study treatment}

In the event of an infusion reaction of grade $\geq 3$ severity during or directly following durvalumab infusion, dosing should be stopped and the patient must permanently discontinue durvalumab treatment.

Study treatment will be permanently stopped in the event of evidence of pregnancy.

In addition, study treatment for any patient may be discontinued for other safety reasons or compliance issues at the discretion of the investigator or sponsor. A patient may choose to discontinue study treatment or study participation at any time for any reason.

A patient who permanently discontinued durvalumab treatment should continue follow-up in the study without additional treatment until progression of disease, completion of all study assessments, or closure 
of the study.

\section{Management of Infusion/Allergic/Hypersensitivity Reactions}

Acute infusion reactions are defined as any $A E$ that occurs during the infusion or within 2 hours after the infusion is completed. Emergency equipment and medication for the treatment of these potential adverse effects (e.g. antihistamines, bronchodilators, intravenous saline, corticosteroids, paracetamol and/or adrenaline] must be available for immediate use. Infusion reactions must be reported as AEs and graded according to the NCl-CTCAE version 4.03 grading scale.

\section{Termination of the Infusion}

The infusion should be terminated and NOT restarted if any of the following AEs occur: anaphylaxis, laryngeal/pharyngeal oedema, severe bronchospasm, chest pain, seizure, severe hypotension

\section{Strategies to improve adherence to interventions $\{11 \mathrm{c}\}$}

Regular review by the treating medical team (Medical and radiation oncology] and manage any toxicities early. Adjuvant phase of immunotherapy following completion of chemoradiation will require careful coordination and support to overcome logistical difficulties particularly for patients from out of town.

\section{Relevant concomitant care permitted or prohibited during the trial $\{11 \mathrm{~d}\}$}

Standard concomitant care to support patients through CRIO will be allowed such as standard antiemetics for cisplatin and appropriate supportive care for patients undergoing chemoradiotherapy.

\section{Provisions for post-trial care $\{30\}$}

Patients will be followed up for a total of 5 years from the time of diagnosis (minimum 2 monthly in the first year, 3 monthly in the second year, and 6 monthly from years 3-5). Patients' treating general practitioners and community will also be engaged in ongoing care particularly in the immediate four to six weeks after chemoradiation when ongoing skin dressings may still be required on a frequent basis.

\section{Outcomes $\{12\}$}

Primary outcome

1. To assess Safety of CRIO: Toxicity, Adverse Events / Serious Adverse Events Reporting

All treatment related toxicities will be recorded at each clinic visit for a total of two years in the Case Report Form using the CTCAE 4.03.

2. To assess the rate of complete response

\section{Patients with measurable disease}

PET scan +/- CT/MRI should be repeated at $12(+/-2)$ weeks. Where results are equivocal, the clinician should either biopsy the suspected residual disease or repeat the imaging after an appropriate interval 
(often 4-6weeks). The patient has achieved a CR if clinical and image findings are negative; if residual image findings are biopsy-negative; or if repeat imaging demonstrates resolution/stability of residual abnormalities.

Once a CR has been declared, patients should continue with the Durvalumab and clinical follow-up at four weekly intervals for a total of 48 weeks from the start of treatment. Physical examination will include direct inspection and palpation of accessible sites. Imaging will be done if there is clinical suspicion of recurrence or progression. Biopsy or cytological confirmation of recurrent disease should be obtained whenever possible. The clinician will record disease status, sites and dates of any relapse and late effects of treatment

Patients who do not achieve a CR should be considered for salvage surgery. Patients who achieve CR after such surgery will be designated $\mathrm{CR}(\mathrm{Su})$ and will undergo follow up as above. Patients with residual disease which is unresectable will be recorded as having a treatment failure.

\section{Patients with large nerve perineural spread}

These patients are not eligible for assessment of complete response and will be assessed as progressionfree or not based on symptoms and/or image findings. Clinical follow-up should occur as above. The first progress MRI should be performed at 3 months from completion of treatment and then 6 monthly thereafter.

\section{Definition of Treatment Failure}

The patient will be considered as having treatment failure if:

- Progressive disease at any time;

- Residual disease at 12 weeks and not amenable to salvage surgery;

- Recurrent disease within the irradiated field during follow up that is biopsy-proven or increases over serial imaging/clinical examination;

- Metastatic disease

Secondary Outcome include overall Survival, progression free survival, and locoregional control will be assessed.

\section{Participant timeline $\{13\}$}

A timeline of 2 years is used to accrue 15 patients. Please also see Fig. 2. Schedule of assessments.

\section{Sample size $\{14\}$}

Fifteen patients would be recruited in the first stage and if 7 or fewer patients respond the trial will be terminated and we will conclude that the treatment is not more effective than standard treatment.

\section{Recruitment \{15\}}


Participants will be identified through specialist referral and through oncology multidisciplinary team meetings. Plastic surgeons and head and neck surgeons are well informed of the trial in our institution as most referrals would come from them. We will also advocate for patients to be treated with CRIO if surgery entails mutilating surgery such as cranio-facial resection which will result in significant functional and cosmetic morbidities.

\section{Assignment Of Interventions: Allocation}

\section{Sequence generation $\{16 a\}$}

Not applicable. All patients in this trial will receive the same treatment.

\section{Concealment mechanism $\{16 \mathrm{~b}\}$}

Not applicable. All patients will receive the same treatment.

\section{Implementation \{16c\}}

Not applicable. All patients will receive the same treatment.

\section{Assignment Of Interventions: Blinding}

\section{Who will be blinded $\{17 \mathrm{a}\}$}

Not applicable. All patients will receive the same treatment.

\section{Procedure for unblinding if needed $\{17 \mathrm{~b}\}$}

Not applicable. All patients will receive the same treatment.

\section{Data Collection And Management}

\section{Plans for assessment and collection of outcomes $\{18 \mathrm{a}\}$}

As per schedule of assessments (Fig. 2). All treatment related toxicities will be recorded at each clinic visit for a total of two years in the Case Report Form using the CTCAE 4.03.

(https://evs.nci.nih.gov/ftp1/CTCAE/CTCAE_4.03/CTCAE_4.03_2010-06-14_QuickReference_8.5× 11.pdf)Disease status of patients will be recorded at each visit during and after completion of treatment. Plans to promote participant retention and complete follow-up $\{18 \mathrm{~b}\}$

Prior to study entry screening will be conducted by trial staff and will include consideration of the ability for the participant to complete treatment and follow-up. A participant is free to withdraw from this project at any stage and have the option to request for trial biological samples to be disposed of.

\section{Data management $\{19\}$}

Only re-identifiable trial data will be stored in a password protected trial database. Trained, registered personnel will have access to the trial database via password protected login. All hardcopy (paper] data will be kept behind locked doors or in locked cabinets in the trial coordinator's room. 


\section{Patient Confidentiality}

The Investigator must ensure that the patient's privacy is maintained. A patient should only be identified by the unit code and his / her patient identifier on the case report forms or other documents submitted to the Data Agency. Only re-identifiable trial data will be stored.

\section{Plans for collection, laboratory evaluation and storage of biological specimens for genetic or molecular analysis in this trial/future use $\{33\}$}

Translational studies will include analyses of tumour tissue and peripheral blood samples from participants (the biospecimens). This include formalin-fixed, paraffin-embedded tumour tissue obtained at baseline (pre-treatment) and peripheral blood before and/or after treatment. Patients will be invited to undergo a repeat core biopsy of the gross disease at 2 weeks into the treatment. Participation for the repeat biopsy is optional. The rationale for the repeat biopsy is to explore if there are any interim biomarkers with treatment response. Peripheral blood will be transported to the central laboratory within 1 hour of collection and processed with serum and peripheral blood mononuclear cells stored for future analysis when trial is complete. Immune markers of interest will be assessed via multiplex immunohistochemistry for formalin-fixed, paraffin-embedded tumour samples or flow cytometry on peripheral blood mononuclear cells. The peripheral blood mononuclear cells will be stored in liquid nitrogen.

\section{Statistical Methods}

\section{Statistical methods for primary and secondary outcomes $\{20 \mathrm{a}\}$}

The study will attempt to recruit all eligible patients who present over 2 years. The actual number will be determined by the frequency of this presentation. Our target accrual will be 15 patients. Our estimate of the complete response rate is $70 \%$, compared to the observed response of $50 \%$ using the same treatment protocol without the addition of durvalumab. A sample size of 15 achieves $52 \%$ power to detect a difference in proportions of 0.20 using a one-sided binomial test with a significance level of 0.06 . Although the study will have limited power to detect a difference in the proportions of patients responding to the treatment protocol compared to the standard of care, the study will provide the first evidence of the effectiveness of CRT and durvalumab in advanced cutaneous SCC, so the information revealed by the study will be valuable.

The analysis will focus on descriptive statistics. The proportion of patients experiencing the primary outcome (complete response] will be presented with 95\% confidence intervals and compared to the hypothesized null effect of 0.5 using a chi-squared test. Categorical variables (Toxicities) will be summarised using frequencies and percentages and continuous variables will be summarised using means with standard deviations or as medians with interquartile ranges. 
Secondary outcomes including overall survival, disease free survival, time to progression and locoregional control will be presented using a Kaplan-Meier curve.

Interim analyses $\{21 b\}$

No interim analysis is planned. The sample size in this study is small.

Methods for additional analyses (e.g. subgroup analyses] \{20b\}

No additional analysis is planned.

\section{Methods in analysis to handle protocol non-adherence and any statistical methods to handle missing data $\{20 \mathrm{c}\}$}

The primary analysis will be intention-to treat, with all patients included, regardless of adherence to the protocol. A per protocol analysis will be conducted as a sensitivity analysis for the proportion of patients showing CR.

\section{Plans to give access to the full protocol, participant level-data and statistical code $\{31 \mathrm{c}\}$}

Not applicable. The protocol is being submitted for consideration of publication. The results of the trial, when completed, will be reported and submitted for publication in appropriate peer reviewed journals.

\section{Oversight And Monitoring}

\section{Composition of the coordinating centre and trial steering committee $\{5 \mathrm{~d}\}$}

Metro North hospital and health service (Queensland, Australia) has the overall responsibility to initiate and conduct the CRIO study and has designated responsibility to the Principal Investigator for the day-today conduct of the study and to lead, train and supervise study site staff. Astra Zeneca will collaborate to support the study as detailed in the research agreement.

This trial is reviewed by a data safety monitoring committee every 6 months to review safety and efficacy aspects of the trial,

Composition of the data monitoring committee, its role and reporting structure $\{21 \mathrm{a}\}$

This study has been reviewed and approved by the local institution's Human Research Ethics Committee (HREC) and will be carried out according to the National Statement on Ethical Conduct in Human Research (2007). It is registered with the Therapeutic Goods Administration, under the Clinical Trial Notification scheme and will be conducted with reference to Guidance on Good Clinical Practice. This trial is also listed on the Royal Brisbane Hospital Risk Register and will be reviewed by a data safety monitoring committee every 6 months to review safety and efficacy of the trial.

All the above groups are independent of the sponsor and have no competing interests.

Any recommendations from the safety committee, annual reports and all SAE will be reported to the local institution's HREC . 
This trial is reviewed by a data safety monitoring committee every 6 months to review safety and efficacy aspects of the trial.

Any recommendations from the safety committee, annual reports and all SAE will be reported to the local institution's HREC.

\section{Frequency and plans for auditing trial conduct $\{23\}$}

This trial is listed on the Royal Brisbane Hospital Risk Register and will be reviewed by a data safety monitoring committee every 6 months to review safety and efficacy of the trial.

Any recommendations from the safety committee, annual reports and all SAE will be reported to the local institution's HREC.

The Investigator will be responsible for assuring that continuing review (at least once per year) of the study is performed by the HREC throughout the duration of the study.

\section{Plans for communicating important protocol amendments to relevant parties (e.g. trial participants, ethical committees] \{25\}}

Approval will be obtained from the local institution's HREC for any protocol amendments and amendments to the information and consent form for this study. Any amendments to the information and consent form also require the study participant to reconsent to participating in the trial.

\section{Dissemination Plans \{31a\}}

The study results based on the trial data will be released to the participating physicians, referring physicians, patients and the general medical community. During study close-out, an interim period will be used to complete data collection, following which the manuscript(s) based on the trial results will be submitted to peer-reviewed journals. Authorship criteria as defined by the International Committee of Medical Journal Editors will be followed.

\section{Discussion}

Patients with advanced cSCC in the head and neck region often require morbid deforming cranio-facial surgeries which often entail orbital exenteration and/or rhinectomy and/or complete facial nerve sacrifice. We have published the outcome of a similar patient cohort treated with definitive chemoradiation which reported $50 \%$ rate of complete response [10]. By eliminating the need for aggressive mutilating surgery, patients were able to live a more normal life with better functional and cosmetic outcome. Our hypothesis is that the addition of durvalumab will improve the rate of complete response to $70 \%$.

This study also aims to explore potential molecular markers in identifying early responders or prognosticators. CXCR4-PET and multiplex immunohistochemical tests were built in to identify potential excellent responders to CRIO. 


\section{Trial Status}

Protocol version 1, dated 3 September 2018. The first patient was accrued on 09/05/2019. It is anticipated that recruitment will be completed in February 2022 although this timeline will be reviewed on a regular basis.

\section{Abbreviations}

cSCC: cutaneous Squamous cell carcinoma; CRIO: ChemoRadiation and ImmuNotherapy; AE: adverse event; ECOG: Eastern Cooperative Oncology Group; NCI CTCAE: National Cancer Institute Common Terminology Criteria for Adverse Events; PD-1: Programmed Death-1; PD-L1: Programmed Death-Ligand 1; CR: complete response; SAE: serious adverse event; SUSAR: suspected unexpected serious adverse reaction; TRAE: treatment related adverse event; UICC/AJCC: Union for International Cancer Control/; GTV: Gross Target Volume; CTV: Clinical Target Volume; PTV: Planning Target Volume; MRI: Magnetic Resonance Imaging; PNS: PeriNeural Spread; HREC: Human Research Ethics Committee

\section{Declarations}

\section{Acknowledgements}

Nil

\section{Authors' contributions $\{31 \mathrm{~b}\}$}

$\mathrm{CL}$ developed the original protocol, obtained ethics approval, writing of the manuscript, and accrue patients to the trial.

TB contributed to the writing of the manuscript

$\mathrm{MN}$ contributed to the development of the protocol and accrual of patients

$\mathrm{KH}$ advised and contributed to the statistics section of the protocol

$\mathrm{BC}$ contributed to the development of the protocol and accrual of patients

LK contributed to the development of the protocol and accrual of patients

PT contributed to the development of the protocol and accrual of patients

MT contributed to the development of the protocol

JK contributed to the development of the protocol, writing of the manuscript, and ethics approval

TL contributed to the development of the protocol and ethics approval 
JE contributed to the development of the protocol and ethics approval

$\mathrm{BH}$ contributed to the development of the protocol, writing of the manuscript, and accrual of patients

\section{Funding $\{4\}$}

Astra Zeneca provided study drug and funding of \$211 000.15 (+ GST). The funding body does not have a role in the collection, analysis and interpretation of data and writing of manuscript. The principal investigator however, need to provide a copy of any publications from CRIO to the funding body at least 30 days prior to publication.

\section{Availability of data and materials $\{29\}$}

The datasets used and/or analysed during the current study are available from the corresponding author on reasonable request.

\section{Ethics approval and consent to participate $\{24\}$}

This study has been reviewed and approved by the RBWH HREC (EC00172).

\section{Consent for publication $\{32\}$}

Not applicable. There is no individual person's data in any form in this manuscript.

\section{Competing interests $\{28\}$}

The authors declare that they have no competing interests.

\section{Author details}

Charles Lin ${ }^{1,5}$ developed the original protocol, obtained ethics approval, writing of the manuscript, and accrue patients to the trial.

Trishna Ballah ${ }^{1,5}$ contributed to the writing of the manuscript

Michelle Nottage ${ }^{2,5}$ contributed to the development of the protocol and accrual of patients

Karen Hay ${ }^{4}$ advised and contributed to the statistics section of the protocol

Benjamin Chua ${ }^{1,5}$ contributed to the development of the protocol and accrual of patients

Lizbeth Kenny ${ }^{1,5}$ contributed to the development of the protocol and accrual of patients

Paul Thomas ${ }^{3,5}$ contributed to the development of the protocol and accrual of patients 
Michele Teng ${ }^{4}$ contributed to the development of the protocol

Jacqui Keller ${ }^{1}$ contributed to the development of the protocol, writing of the manuscript and ethics approval

Trang Le ${ }^{1}$ contributed to the development of the protocol and ethics approval

Jennifer Edmunds ${ }^{1}$ contributed to the development of the protocol and ethics approval

Brett Hughes ${ }^{2,5}$ contributed to the development of the protocol, writing of the manuscript, and accrual of patients

${ }^{1}$ Department of Radiation Oncology, Royal Brisbane and Women's Hospital, Australia

${ }^{2}$ Department of Medical Oncology, Royal Brisbane and Women's Hospital, Australia

${ }^{3}$ Department of Nuclear Medicine, Royal Brisbane and Women's Hospital, Australia

${ }^{4}$ Queensland institute of Medical Research, Berghofer Medical Research Institute, Australia

${ }^{5}$ Faculty of Medicine, University of Queensland, Australia

\section{References}

1. Veness MJ, Quinn DI, Ong CS, Keogh AM, Macdonald PS, Cooper SG, et al. Aggressive cutaneous malignancies following cardiothoracic transplantation: the Australian experience. Cancer. 1999;85(8]:1758-64.

2. Rowe DE, Carroll RJ, Day CL, Jr. Prognostic factors for local recurrence, metastasis, and survival rates in squamous cell carcinoma of the skin, ear, and lip. Implications for treatment modality selection. J Am Acad Dermatol. 1992;26(6]:976-90.

3. Wang JT, Palme CE, Wang AY, Morgan GJ, Gebski V, Veness MJ. In patients with metastatic cutaneous head and neck squamous cell carcinoma to cervical lymph nodes, the extent of neck dissection does not influence outcome. J Laryngol Otol. 2013;127 Suppl 1:S2-7.

4. Veness MJ, Porceddu S, Palme CE, Morgan GJ. Cutaneous head and neck squamous cell carcinoma metastatic to parotid and cervical lymph nodes. Head Neck. 2007;29(7]:621-31.

5. Veness MJ, Palme CE, Morgan GJ. High-risk cutaneous squamous cell carcinoma of the head and neck: results from 266 treated patients with metastatic lymph node disease. Cancer. 2006;106(11]:2389-96.

6. Guthrie TH, Jr., Porubsky ES, Luxenberg MN, Shah KJ, Wurtz KL, Watson PR. Cisplatin-based chemotherapy in advanced basal and squamous cell carcinomas of the skin: results in 28 patients including 13 patients receiving multimodality therapy. J Clin Oncol. 1990;8(2]:342-6. 
7. Khansur T, Kennedy A. Cisplatin and 5-fluorouracil for advanced locoregional and metastatic squamous cell carcinoma of the skin. Cancer. 1991;67(8]:2030-2.

8. Sadek H, Azli N, Wendling JL, Cvitkovic E, Rahal M, Mamelle G, et al. Treatment of advanced squamous cell carcinoma of the skin with cisplatin, 5-fluorouracil, and bleomycin. Cancer. 1990;66(8]:1692-6.

9. Shin DM, Glisson BS, Khuri FR, Clifford JL, Clayman G, Benner SE, et al. Phase II and biologic study of interferon alfa, retinoic acid, and cisplatin in advanced squamous skin cancer. J Clin Oncol. 2002;20(2]:364-70.

10. Nottage MK, Lin C, Hughes BG, Kenny L, Smith DD, Houston K, et al. Prospective study of definitive chemoradiation in locally or regionally advanced squamous cell carcinoma of the skin. Head Neck. 2017;39(4]:679-83.

11. Pickering CR, Zhang J, Neskey DM, Zhao M, Jasser SA, Wang J, et al. Squamous cell carcinoma of the oral tongue in young non-smokers is genomically similar to tumours in older smokers. Clin Cancer Res. 2014;20(14]:3842-8.

12. Pickering CR, Zhou JH, Lee JJ, Drummond JA, Peng SA, Saade RE, et al. Mutational landscape of aggressive cutaneous squamous cell carcinoma. Clin Cancer Res. 2014;20(24]:6582-92.

13. Muhleisen B, Petrov I, Gachter T, Kurrer M, Scharer L, Dummer R, et al. Progression of cutaneous squamous cell carcinoma in immunosuppressed patients is associated with reduced CD123+ and FOXP3+ cells in the perineoplastic inflammatory infiltrate. Histopathology. 2009;55(1]:67-76.

14. Freeman A, Bridge JA, Maruthayanar P, Overgaard NH, Jung JW, Simpson F, et al. Comparative immune phenotypic analysis of cutaneous Squamous Cell Carcinoma and Intraepidermal Carcinoma in immune-competent individuals: proportional representation of CD8+ T-cells but not FoxP3+ Regulatory T-cells is associated with disease stage. PLoS One. 2014;9(10]:e110928.

15. Euvrard S, Kanitakis J, Pouteil-Noble C, Disant F, Dureau G, Finaz de Villaine J, et al. Aggressive squamous cell carcinomas in organ transplant recipients. Transplant Proc. 1995;27(2]:1767-8.

16. Yu SH, Bordeaux JS, Baron ED. The immune system and skin cancer. Adv Exp Med Biol. 2014;810:182-91.

17. Lippman SM, Parkinson DR, Itri LM, Weber RS, Schantz SP, Ota DM, et al. 13-cis-retinoic acid and interferon alpha-2a: effective combination therapy for advanced squamous cell carcinoma of the skin. J Natl Cancer Inst. 1992;84(4]:235-41.

18. Alexandrov LB, Nik-Zainal S, Wedge DC, Aparicio SA, Behjati S, Biankin AV, et al. Signatures of mutational processes in human cancer. Nature. 2013;500(7463]:415-21.

19. Grob JJ, Mendoza RG, Basset-Seguin N, Vornicova O, Schachter J, Joshi A, et al. LBA72 - Pembrolizumab for recurrent/metastatic cutaneous squamous cell carcinoma (CSCC): Efficacy and safety results from the phase II KEYNOTE-629 study. Annals of Oncology. 2019; 30 Suppl 5: p908

20. Migden MR, Rischin D, Schmults CD, Guminski A, Hauschild A, Lewis KD et al. PD-1 Blockade with Cemiplimab in Advanced Cutaneous Squamous-Cell Carcinoma. N Engl J Med 2018; 379:341-351. 
DOI: 10.1056/NEJMoa1805131

21. Antonia SJ, Villegas A, Daniel D, Vicente D, Murakami S, Hui R et al. Overall Survival with Durvalumab after Chemoradiotherapy in Stage III NSCLC. N Engl J Med 379:2342-2350. DOI:

10.1056/NEJMoa1809697

22. Beavis PA, Milenkovski N, Henderson MA, John LB, Allard B, Loi S, et al. Adenosine Receptor 2A Blockade Increases the Efficacy of Anti-PD-1 through Enhanced Antitumour T-cell Responses. Cancer Immunol Res. 2015;3(5]:506-17.

23. Stagg J, Smyth MJ. Extracellular adenosine triphosphate and adenosine in cancer. Oncogene. 2010;29(39]:5346-58.

24. Young A, Mittal D, Stagg J, Smyth MJ. Targeting cancer-derived adenosine: new therapeutic approaches. Cancer Discov. 2014;4(8]:879-88.

25. Stagg J, Divisekera U, McLaughlin N, Sharkey J, Pommey S, Denoyer D, et al. Anti-CD73 antibody therapy inhibits breast tumour growth and metastasis. Proc Natl Acad Sci U S A. 2010;107(4]:154752.

26. Stagg J, Divisekera U, Duret H, Sparwasser T, Teng MW, Darcy PK, et al. CD73-deficient mice have increased antitumour immunity and are resistant to experimental metastasis. Cancer Res. 2011;71(8]:2892-900.

27. Beavis PA, Divisekera U, Paget C, Chow MT, John LB, Devaud C, et al. Blockade of A2A receptors potently suppresses the metastasis of CD73+ tumours. Proc Natl Acad Sci U S A. 2013;110(36]:14711-6.

28. Young A, Ngiow SF, Barkauskas DS, Sult E, Hay C, Blake SJ, et al. Co-inhibition of CD73 and A2AR Adenosine Signaling Improves Anti-tumour Immune Responses. Cancer Cell. 2016;30(3]:391-403.

29. Mittal D, Young A, Stannard K, Yong M, Teng MW, Allard B, et al. Antimetastatic effects of blocking PD-1 and the adenosine A2A receptor. Cancer Res. 2014;74(14]:3652-8.

30. Loi S, Pommey S, Haibe-Kains B, Beavis PA, Darcy PK, Smyth MJ, et al. CD73 promotes anthracycline resistance and poor prognosis in triple negative breast cancer. Proc Natl Acad Sci U S A. 2013;110(27]:11091-6.

31. Allard B, Pommey S, Smyth MJ, Stagg J. Targeting CD73 enhances the antitumour activity of antiPD-1 and anti-CTLA-4 mAbs. Clin Cancer Res. 2013;19(20]:5626-35.

32. Feig C, Jones JO, Kraman M, Wells RJ, Deonarine A, Chan DS, et al. Targeting CXCL12 from FAPexpressing carcinoma-associated fibroblasts synergizes with anti-PD-L1 immunotherapy in pancreatic cancer. Proc Natl Acad Sci U S A. 2013;110(50]:20212-7.

33. Gourni E, Demmer O, Schottelius M, D'Alessandria C, Schulz S, Dijkgraaf I, et al. PET of CXCR4 expression by a (68]Ga-labeled highly specific targeted contrast agent. J Nucl Med. 2011;52(11]:1803-10.

34. Journal of the ICRU Vol 10 No 1 (2010) Report 83 The International Commission on Radiation Units and Measurements doi:10.1093/jicru/ndq001 
35. Gregoire V, Ang K, Budach W, Grau C, Hamoir M, Langendijk JA, et al. Delineation of the neck node levels for head and neck tumours: a 2013 update. DAHANCA, EORTC, HKNPCSG, NCIC CTG, NCRI, RTOG, TROG consensus guidelines. Radiother Oncol. 2014;110(1]:172-81. 37. Alexandrov et al. 2013

\section{Figures}

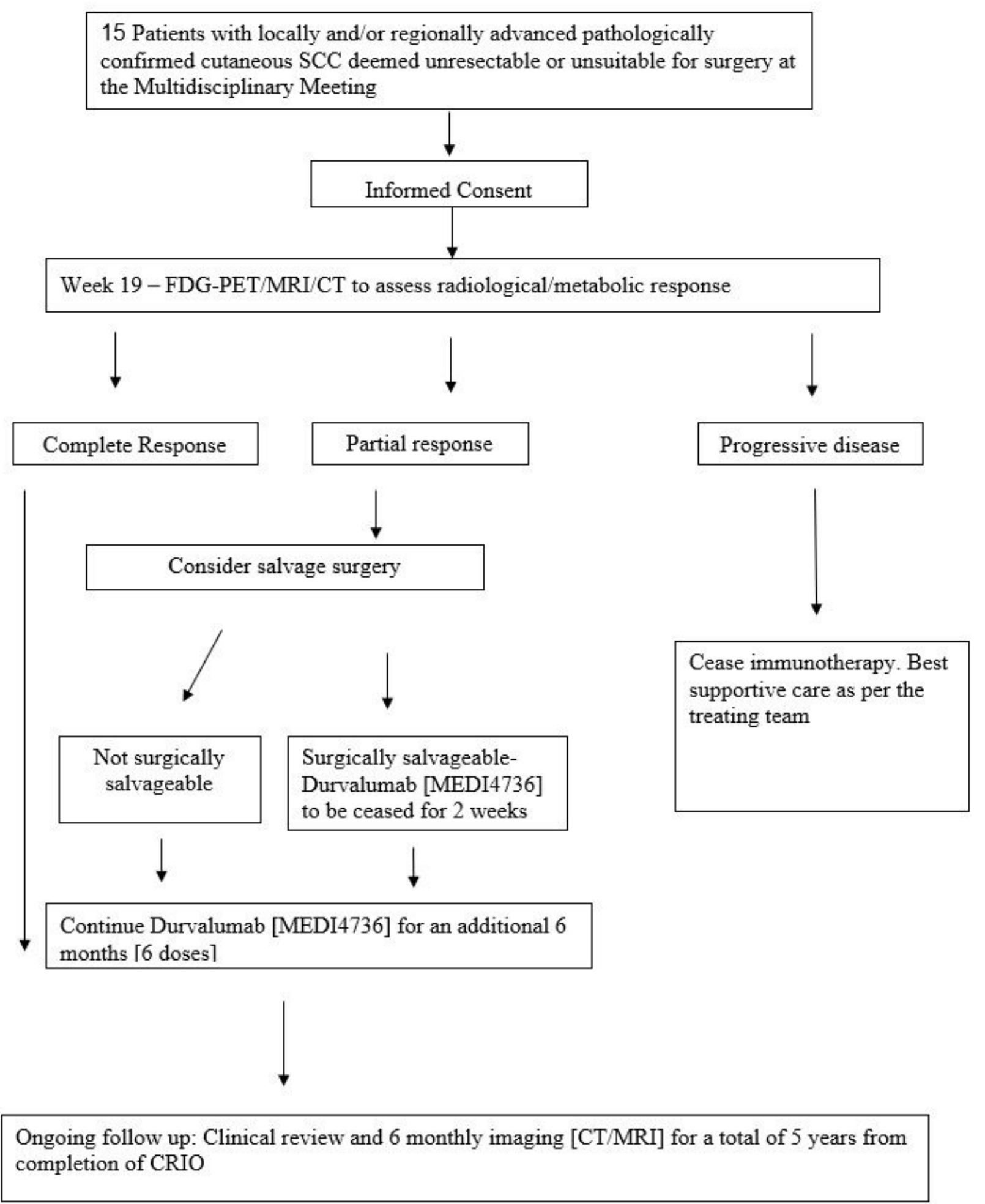


Consort diagram of CRIO trial

\begin{tabular}{|c|c|c|c|c|c|c|c|c|c|c|c|c|c|c|c|}
\hline Investigation & $\begin{array}{l}\text { Screening } \\
\text { Baseline }\end{array}$ & Wk 1 & Wk2 & Wk3 & Wk4 & Wk5 & Wk6 & Wk7 & Wk9 & Wk13 & Wk17 & $\begin{array}{l}\text { Wk } \\
19\end{array}$ & $\begin{array}{l}\text { Wk } 21,25, \\
29,33,37, \\
41\end{array}$ & Wk 27 & $\begin{array}{l}\text { Months post treatment } \\
2,4,6,8,10,12,15,18,21 \\
24,30,36,42,48,54,60\end{array}$ \\
\hline Informed consent & $\mathrm{x}$ & & & & & & & & & & & & & & \\
\hline $\begin{array}{l}\text { Histological } \\
\text { confirmation of SCC }\end{array}$ & $\mathrm{x}$ & & & & & & & & & & & & & & \\
\hline $\begin{array}{l}\text { Repeat biopsy (skin or } \\
\text { lymph nodes] }\end{array}$ & & & $\mathrm{x}^{1}$ & & & & & & & & & & & & \\
\hline Medical history & $x^{6}$ & & & & & & & & & & & & & & \\
\hline $\begin{array}{l}\text { Physical examination } \\
\text { (ECOG, vital signs, } \\
\text { toxicity] }\end{array}$ & $x^{6}$ & $\mathrm{x}$ & $\mathrm{x}$ & $\mathrm{x}$ & $\mathrm{x}$ & $\mathrm{x}$ & $\mathrm{x}$ & $\mathrm{x}$ & $\mathrm{x}$ & $\mathrm{x}$ & $\mathrm{x}$ & & $\mathrm{x}$ & & $\mathrm{x}$ \\
\hline Concomitant meds & $\mathrm{x}$ & & & & & & & & & & & & & & \\
\hline Urine analysis & $\mathrm{x}$ & & & & & & & & & & & & & & \\
\hline Pregnancy test & $x^{6}$ & & & & & & & & & & & & & & \\
\hline FBC & $x^{6}$ & $\mathrm{x}$ & $\mathrm{x}$ & $\mathrm{x}$ & $\mathrm{x}$ & $\mathrm{x}$ & $\mathrm{x}$ & $\mathrm{x}$ & $\mathrm{x}$ & $\mathrm{x}$ & $\mathrm{x}$ & & $\mathrm{x}$ & & $\mathrm{x}^{4}$ \\
\hline U\&E, LFT's & $x^{6}$ & $\mathrm{x}$ & $\mathrm{x}$ & $\mathrm{x}$ & $\mathrm{x}$ & $\mathrm{x}$ & $\mathrm{x}$ & $\mathrm{x}$ & $\mathrm{x}$ & $\mathrm{x}$ & $\mathrm{x}$ & & $\mathrm{x}$ & & $\mathrm{x}^{4}$ \\
\hline $\begin{array}{l}\text { Coagulation profile, } \\
\text { TSH, hep, HIV }\end{array}$ & $\mathrm{x}$ & & & & & & & & & & & & & & \\
\hline FDG PET & $x^{7}$ & & & & & & & & & & & $\mathrm{x}$ & & $\mathrm{x}^{2}$ & \\
\hline CXCR4 PET /CT & $\mathrm{x}^{3}$ & & & $\mathrm{x}$ & & & & & & & & & & & \\
\hline Contrast CT / MRI & $\mathrm{x}^{7}$ & & & & & & & & & & & $\mathrm{x}^{4}$ & & & \\
\hline Disease status & & $\mathrm{x}$ & $\mathrm{x}$ & $\mathrm{x}$ & $\mathrm{x}$ & $\mathrm{x}$ & $\mathrm{x}$ & $\mathrm{x}$ & $\mathrm{x}$ & $\mathrm{x}$ & $\mathrm{x}$ & & $\mathrm{x}$ & & $\mathrm{x}$ \\
\hline Blood & $\mathrm{x}$ & & & $\mathrm{x}$ & & & & & & $\mathrm{x}$ & & & $\mathrm{x}^{5}$ & & \\
\hline \multicolumn{16}{|c|}{$=$ Not mandatory } \\
\hline$=$ if week 19 FDG PET is equi & & & & ${ }^{6}=\mathrm{d}$ & within & days of $t$ & al registra: & & & & & & & & \\
\hline$=$ pretreatment & & & & ${ }^{7}=$ & within & days of $t$ & al registra & & & & & & & & \\
\hline
\end{tabular}

${ }^{4}=$ if clinically indicated

Patients who are considered treatment failures will be followed at the above time points for survival only. This may be done at clinic visits when seen for ongoing treatment or by telephone call.

\section{Figure 2}

Schedule of Assessments 